\title{
Stability Analysis of the Multi-Objective Multi-Guided Particle Swarm Optimizer
}

\author{
Christopher W. Cleghorn, Christiaan Scheepers and Andries P. Engelbrecht
}

\author{
Department of Computer Science \\ University of Pretoria \\ Pretoria, South Africa \\ \{ccleghorn, engel\}@cs.up.ac.za, cscheepers@acm.org
}

\begin{abstract}
At present particle swarm optimizers (PSO) designed for multi-objective optimization have undergone no form of theoretical stability analysis. This paper derives the sufficient and necessary conditions for order-1 and order-2 stability of the recently proposed multi-guided PSO (MGPSO), which was designed specifically for multi-objective optimization. The paper utilizes a recently published theorem for performing stability analysis on PSO variants, which requires minimal modeling assumptions. It is vital for PSO practitioners to know the actual criteria for particle stability of the given PSO variant being used, as it been shown that particle stability has a considerable impact on PSO's performance. This paper empirically validates its theoretical findings by comparing the derived stability criteria against those of an assumption free MGPSO algorithm. It was found that the derived criteria for order- 1 and order-2 stability are an accurate predictor of the unsimplified MGPSO's particle behavior.
\end{abstract}

\section{Introduction}

Recently, a particle swarm optimizer (PSO) variant, the multi-guided PSO (MGPSO) was proposed for multi-objective optimization [14,15]. It was found that MGPSO was highly competitive to the current state of the art PSO based multiobjective optimization algorithms, such as speed-constrained multi-objective particle swarm optimization (SMPSO) [10], optimized multi-objective particle swarm optimization (OMOPSO) [13], and the vector evaluated particle swarm optimizer (VEPSO) $[11,12]$. Furthermore, the MGPSO was also shown to be highly competitive with the current state of the art evolutionary multiple objective optimizers, such as the non-dominated sorting genetic algorithm II (NSGAII) [8], strength Pareto evolutionary algorithm 2 (SPEA2) [18], pareto envelopebased selection algorithm II (PESA-II) [7], and the multi-objective evolutionary algorithm based on decomposition (MOEA/D) [17].

With the introduction of any new optimization algorithm comes an array of unknown algorithm characteristics to be understood. The characteristic that this paper focuses on is particle stability. It has been empirically shown that 
order-1 and order- 2 particle stability has a considerable impact on performance [4]. Furthermore, it was shown that parameter configurations that resulted in particle instability almost always caused PSO to perform worse than random search [4]. The clear relationship between PSO particle stability and the algorithm's performance, implies that knowing the criteria for particle stability is needed for effective use of a PSO variant.

Given that MGPSO has a similar structure to that of the original PSO [9] with the presence of inertia as proposed by Shi [16], existing PSO theory can be readily applied to the stability analysis of MGPSO. Specifically, Cleghorn and Engelbrecht [6] recently proposed a general theorem for deriving stability criteria for a class of PSO variants under minimal modeling assumptions. To the authors' knowledge this is the first paper to perform stability analysis of a multiobjective PSO. The theoretically derived region for particle stability of MGPSO is also empirically validated utilizing the assumption for free methodology for stability region validation, as presented in $[1,3]$, and used in $[2,5]$.

A description of MGPSO is provided in section 2. The theoretical derivation of the order-1 and order-2 stable regions of MGPSO are presented in section 3 . The experimental setup and results empirically validating the derived stable regions are presented in sections 4 and 5 respectively. A summary of the findings of this paper are presented in section 6 .

\section{Multi-guided Particle Swarm Optimizer}

The MGPSO algorithm was proposed by Scheepers and Engelbrecht $[14,15]$ and is inspired by the vector evaluated particle swarm optimizer (VEPSO) as proposed by Parsopoulos and Vrahatis [11,12]. MGPSO is a multi-swarm multiobjective PSO variant, where each objective is optimized by a sub-swarm. Similar to VEPSO, the Pareto-optimal solutions are stored in an archive. Scheepers and Engelbrecht proposed that a third attractor be added to the velocity update equation, in addition to the usual social and cognitive attractors. The aim of the new attractor is to pull particles towards the Pareto-optimal front (POF). The third attractor is selected from the archive of non-dominated solutions. The archive attractor is selected from the tournament pool as the one with the largest crowding distance [8] to promote convergence to a diverse pareto-front.

The velocity and position update equations of MGPSO are defined as follows:

$$
\begin{aligned}
\mathbf{v}_{i}(t+1)= & w \mathbf{v}_{i}(t)+c_{1} \mathbf{r}_{1} \otimes\left(\mathbf{y}_{i}(t)-\mathbf{x}_{i}(t)\right)+\lambda_{i} c_{2} \mathbf{r}_{2} \otimes\left(\hat{\mathbf{y}}_{i}(t)-\mathbf{x}_{i}(t)\right) \\
& +\left(1-\lambda_{i}\right) c_{3} \mathbf{r}_{3} \otimes\left(\hat{\mathbf{a}}_{i}(t)-\mathbf{x}_{i}(t)\right) \\
\mathbf{x}_{i}(t+1)= & \mathbf{x}(t)+\mathbf{v}_{i}(t+1),
\end{aligned}
$$

where $\mathbf{r}_{1}, \mathbf{r}_{2}, \mathbf{r}_{3} \sim U(0,1)^{d}$, and $d$ is the dimension of the objective functions PSO. The operator $\otimes$ is used to indicate component-wise multiplication of two vectors. The positions $\mathbf{y}_{i}$ and $\hat{\mathbf{y}}_{i}$ are respectively the "best" positions that particle $i$ and particle $i$ 's neighborhood of particles have visited. In this paper, "best" is defined as the location where a particle has obtained the lowest objective 
function evaluation. The coefficients $c_{1}, c_{2}, c_{3}$, and $w$ are the cognitive, social, archive, and inertia weights respectively. $\lambda_{i}$ is the exploitation trade-off coefficient for particle $i$, is initialized as a random constant sampled uniformly from $(0,1)\left(\lambda_{i}\right.$ does not vary over iterations). The MGPSO algorithm is summarized in algorithm 1 .

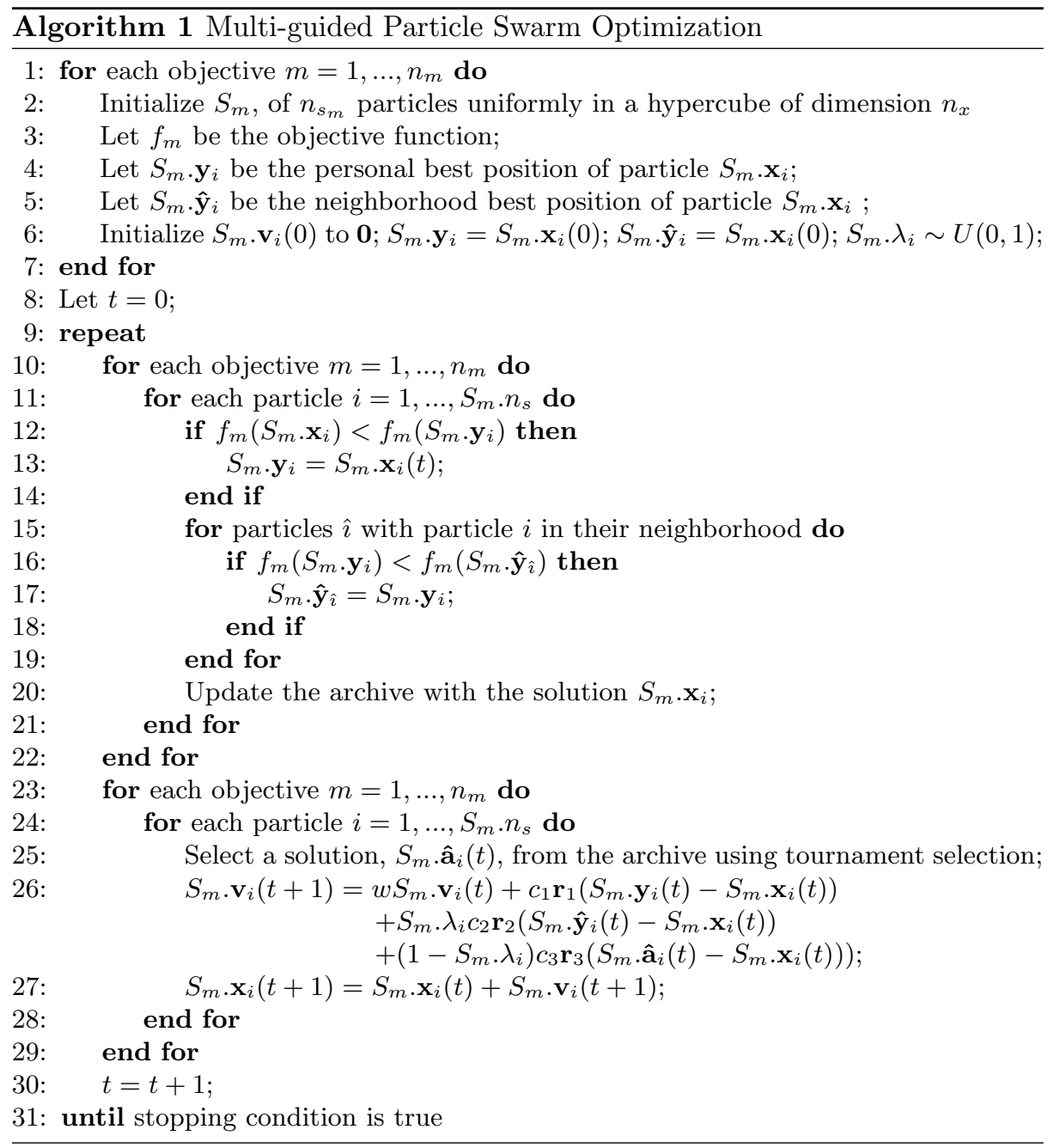

\section{Theoretical Derivation}

This section presents a theoretical derivation of the order- 1 and order- 2 stable regions for the MGPSO algorithm. 
To derive order-1 and order-2 stable regions for MGPSO, the following general theorem of Cleghorn and Engelbrecht [6] is used:

Theorem 1. The following properties hold for all PSO variants of the form:

$$
x_{k}(t+1)=x_{k}(t) \alpha+x_{k}(t-1) \beta+\gamma_{t}
$$

where $k$ indicates the vector component, $\alpha$ and $\beta$ are well defined random variables, and $\left(\gamma_{t}\right)$ is a sequence of well defined random variables. In the context of this work, a random variable is said to be well defined if it has an expectation and a variance.

1. Assuming $\boldsymbol{i}_{t}$ converges, particle positions are order-1 stable for every initial condition if and only if $\rho(\mathbf{A})<1^{1}$, where

$$
\mathbf{A}=\left[\begin{array}{cc}
E[\alpha] & E[\beta] \\
1 & 0
\end{array}\right] \text { and } \boldsymbol{i}_{t}=\left[\begin{array}{c}
E\left[\gamma_{t}\right] \\
0
\end{array}\right]
$$

2. The particle positions are order-2 stable if $\rho(\boldsymbol{B})<1$ and $\left(\boldsymbol{j}_{t}\right)$ converges, where

$$
\boldsymbol{B}=\left[\begin{array}{ccccc}
E[\alpha] & E[\beta] & 0 & 0 & 0 \\
1 & 0 & 0 & 0 & 0 \\
0 & 0 & E\left[\alpha^{2}\right] & E\left[\beta^{2}\right] & 2 E[\alpha \beta] \\
0 & 0 & 1 & 0 & 0 \\
0 & 0 & E[\alpha] & 0 & E[\beta]
\end{array}\right] \text { and } \boldsymbol{j}_{t}=\left[\begin{array}{c}
E\left[\gamma_{t}\right] \\
0 \\
E\left[\gamma_{t}^{2}\right] \\
0 \\
0
\end{array}\right]
$$

under the assumption that the limits of $\left(E\left[\gamma_{t} \alpha\right]\right)$ and $\left(E\left[\gamma_{t} \beta\right]\right)$ exist.

3. Assuming that $x(t)$ is order-1 stable, then the following is a necessary condition for order-2 stability:

$$
\begin{array}{r}
1-E[\alpha]-E[\beta] \neq 0 \\
1-E\left[\alpha^{2}\right]-E\left[\beta^{2}\right]-\left(\frac{2 E[\alpha \beta] E[\alpha]}{1-E[\beta]}\right)>0
\end{array}
$$

4. The convergence of $E\left[\gamma_{t}\right]$ is a necessary condition for order-1 stability, and the convergence of both $E\left[\gamma_{t}\right]$ and $E\left[\gamma_{t}^{2}\right]$ is a necessary condition for order-2 stability.

The MGPSO's update equation (1), can be written in the form of equation (3) by setting:

$$
\begin{aligned}
\alpha & =(1+w)-c_{1} r_{1}-\lambda c_{2} r_{2}-(1-\lambda) c_{3} r_{3}, \quad \beta=-w \\
\gamma_{t} & =c_{1} r_{1} y(t)+\lambda c_{2} r_{2} \hat{y}(t)+(1-\lambda) c_{3} r_{3} \hat{a}(t)
\end{aligned}
$$

In order to utilize theorem 1 , the following modeling assumption is used:

\footnotetext{
${ }^{1} \rho(\mathbf{M})$ denotes the spectral radius of the matrix $\mathbf{M}$
} 
Definition 1. Non-stagnant distribution assumption [6]:

It is assumed that $\hat{\boldsymbol{y}}_{i}(t), \boldsymbol{y}_{i}(t)$, and $\hat{\boldsymbol{a}}_{i}(t)$ are random variables sampled from a time dependent distribution, such that $\hat{\boldsymbol{y}}_{i}(t), \boldsymbol{y}_{i}(t)$, and $\hat{\boldsymbol{a}}_{i}(t)$ have well defined expectations and variances for each $t$ and that $\lim _{t \rightarrow \infty} E\left[\hat{\boldsymbol{y}}_{i}(t)\right], \lim _{t \rightarrow \infty} E\left[\boldsymbol{y}_{i}(t)\right]$, $\lim _{t \rightarrow \infty} E\left[\hat{\boldsymbol{a}}_{i}(t)\right], \lim _{t \rightarrow \infty} V\left[\hat{\boldsymbol{y}}_{i}(t)\right], \lim _{t \rightarrow \infty} V\left[\boldsymbol{y}_{i}(t)\right]$ and $\lim _{t \rightarrow \infty} V\left[\hat{\boldsymbol{a}}_{i}(t)\right]$ exist.

It is clear from part 4 of theorem 1 that the non-stagnant distribution assumption is a necessary condition for order- 1 and order-2 stability. In order to obtain the criteria for order- 1 stability, part 1 of theorem 1 is used. Specifically, the following expectations are required:

$$
\begin{gathered}
E[\alpha]=(1+w)-\frac{c_{1}}{2}-\frac{\lambda c_{2}}{2}-\frac{(1-\lambda) c_{3}}{2}, E[\beta]=-w, \\
E\left[\gamma_{t}\right]=\frac{1}{2}\left(c_{1} E[y(t)]+\lambda c_{2} E[\hat{y}(t)]+(1-\lambda) c_{3} E[\hat{a}(t)]\right) .
\end{gathered}
$$

Given the non-stagnant distribution assumption, it follows by the sum of convergent sequences that $E\left[\gamma_{t}\right]$ converges, and therefore $\mathbf{i}_{t}$ converges. The criteria for order-1 stability is determined by coefficients that satisfy $\rho(\mathbf{A})<1$. After some algebraic manipulation, the following criteria for order-1 stability is obtained:

$$
|w|<1 \text { and } 0<c_{1}+\lambda c_{2}+(1-\lambda) c_{3}<4(w+1),
$$

or in the case of $c=c_{1}=c_{2}=c_{3}$,

$$
|w|<1 \text { and } 0<2 c<4(w+1) .
$$

Part 3 of theorem 1 is used to derive the criteria necessary for order-2 stability. The calculation of additional expected values is needed. In order to calculate $E\left[\alpha^{2}\right], \alpha^{2}$ is first calculated as:

$$
\begin{aligned}
\alpha^{2} & =\left((1+w)-c r_{1}-\lambda c r_{2}-(1-\lambda) c r_{3}\right)^{2} \\
& =(1+w)^{2}-c_{1} r_{1}(1+w)-\lambda c_{2} r_{2}(1+w)-(1+w)(1-\lambda) c_{3} r_{3} \\
& -c_{1} r_{1}(1+w)+c_{1}^{2} r_{1}^{2}+\lambda c_{1} c_{2} r_{1} r_{2}+(1-\lambda) c_{1} c_{3} r_{1} r_{3} \\
& -\lambda c_{2} r_{2}(1+w)+\lambda c_{1} c_{2} r_{1} r_{2}+\lambda^{2} c_{2}^{2} r_{2}^{2}+\lambda(1-\lambda) c_{2} c_{3} r_{2} r_{3} \\
& -(1+w)(1-\lambda) c_{3} r_{3}+(1-\lambda) c_{1} c_{3} r_{1} r_{3}+\lambda(1-\lambda) c_{2} c_{3} r_{2} r_{3}+(1-\lambda)^{2} c_{3}^{2} r_{3}^{2}
\end{aligned}
$$

Applying the expectation operator results in

$$
\begin{aligned}
E\left[\alpha^{2}\right] & =(1+w)^{2}-\frac{c_{1}}{2}(1+w)-\lambda \frac{c_{2}}{2}(1+w)-(1+w)(1-\lambda) \frac{c_{3}}{2} \\
& -\frac{c_{1}}{2}(1+w)+\frac{c_{1}^{2}}{3}+\lambda \frac{c_{1} c_{2}}{4}+(1-\lambda) \frac{c_{1} c_{3}}{4} \\
& -\lambda \frac{c_{2}}{2}(1+w)+\lambda \frac{c_{1} c_{2}}{4}+\lambda^{2} \frac{c_{2}^{2}}{3}+\lambda(1-\lambda) \frac{c_{2} c_{3}}{4} \\
& -(1+w)(1-\lambda) \frac{c_{3}}{2}+(1-\lambda) \frac{c_{1} c_{3}}{4}+\lambda(1-\lambda) \frac{c_{2} c_{3}}{4}+(1-\lambda)^{2} \frac{c_{3}^{2}}{3}
\end{aligned}
$$


Let $c=c_{1}=c_{2}=c_{3}$, then after some algebraic manipulation, equation (11) becomes

$$
\begin{aligned}
E\left[\alpha^{2}\right] & =(1+w)^{2}-c(1+w)-\lambda c(1+w)-(1+w)(1-\lambda) c \\
& +c^{2}\left(\frac{1}{3}+\frac{\lambda}{2}+\frac{1-\lambda}{2}+\frac{\lambda^{2}}{3}+\frac{\lambda(1-\lambda)}{2}+\frac{(1-\lambda)^{2}}{3}\right) \\
& =(1+w)((1+w)-2 c)+\frac{c^{2}}{6}\left(\lambda^{2}-\lambda+7\right)
\end{aligned}
$$

The following expectations are also needed:

$$
E[\alpha \beta]=-w E[\alpha]=-w((1+w)-c) \text { and } E\left[\beta^{2}\right]=w^{2}
$$

In order to derive the criteria necessary for order-2 stability, first consider the condition of equation (6) in part 3 of theorem 1 :

$$
1+E[\alpha]+E[\beta] \neq 0 \Longrightarrow c_{1}+\lambda c_{2}+(1-\lambda) c_{3} \neq 0
$$

or if $c=c_{1}=c_{2}=c_{3}$, simply $c \neq 0$.

Now consider the condition of equation (7) in part 3 of theorem 1:

$$
\begin{array}{r}
1-E\left[\alpha^{2}\right]-E\left[\beta^{2}\right]-\left(\frac{2 E[\alpha \beta] E[\alpha]}{1-E[\beta]}\right)>0 \\
\Longrightarrow 2 c-2 w c+\left(\frac{2 w c^{2}}{(1+w)}\right)-\frac{c^{2}}{6}\left(\lambda^{2}-\lambda+7\right)>0
\end{array}
$$

Solving equation (14) as a quadric form equal to 0 leads to

$$
c<\frac{12\left(1-w^{2}\right)}{\left(\lambda^{2}-\lambda+7\right)(w+1)-12 w}
$$

Merging the conditions for order-2 stability in equations (15) and (13) with the conditions for order-1 stability of equation (9) leads to the following criteria for order-1 and order-2 stability:

$$
0<c<\frac{12\left(1-w^{2}\right)}{\left(\lambda^{2}-\lambda+7\right)(w+1)-12 w}, \quad|w|<1
$$

This merger is possible because the region defined by equation (15) is a subset of the region defined by equation (9). The conditions derived for order-2 stability are only the necessary conditions. To verify that they are sufficient, part 2 of theorem 1 is used: Given the complexity of symbolically solving $\rho(\boldsymbol{B})<1$, an empirical approach is utilized in line with that used by Cleghorn and Engelbrecht [6]. The experimental procedure followed is: $10^{9}$ random configurations of the form $\{w, c, \lambda\}$ were generated such that equations (13) and (15) were satisfied. It was then tested if the condition, $\rho(\boldsymbol{B})<1$, was satisfied or not. In all of the cases it was found, that if equations (13) and (15) were satisfied, then the condition 
$\rho(\boldsymbol{B})<1$ held. This finding is strong evidence that the criteria of equation (16) are both sufficient and necessary for order-1 and order-2 stability.

The manner in which $\lambda$ affects the stability region is illustrated in figure 1. The closer $\lambda$ gets to 0.5 , the more the apex of the stability region extends. As $\lambda$ approaches either 0 or 1 from 0.5 the stability region reduces in size in a symmetric manner. Given that $\lambda$ may be initialized to any value in the range $(0,1)$, selecting coefficients such that equation (16) is satisfied for $\lambda=0$ (or $\lambda=1$ ) will ensure that every particle will be both order- 1 and order- 2 regardless of the particle specific $\lambda$.

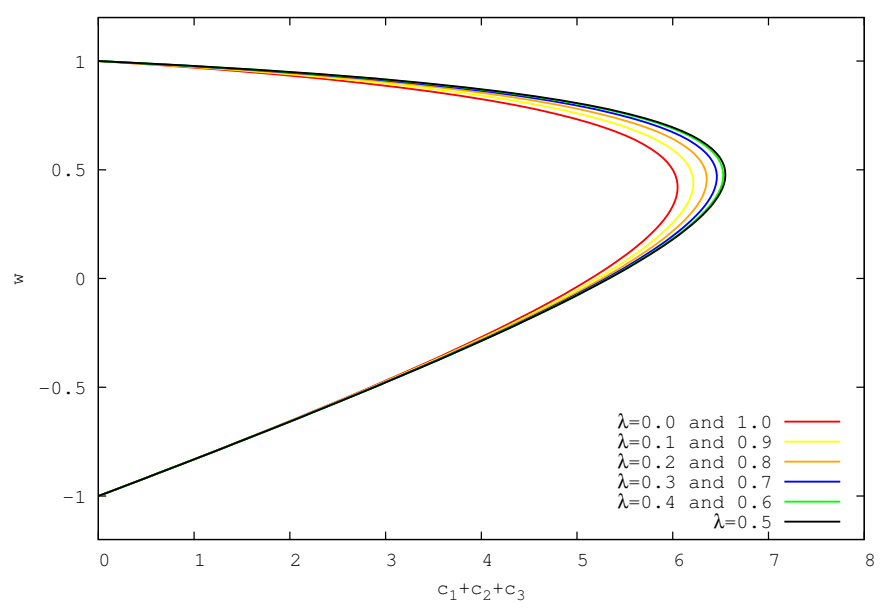

Fig. 1: MGPSO convergent regions for $\lambda=0,0.1,0.2,0.3,0.4,0.5,0.6,0.7,0.8$, 0.9 , and 1

While the modeling assumption utilized in the section is minimal, it is still required to confirm whether or not the newly derived stability criteria are truly representative of the unsimplified MGPSO's behaviour. This is done in the next section.

\section{Empirical Setup}

This section utilizes the method for empirically validating the stability region of PSO variants as proposed by Cleghorn and Engelbrecht $[1,3]$.

A swarm size of 64 particles per objective, and 5000 iterations are used in the experiment. Two objective functions where considered. Particle velocities were initialized to $\mathbf{0}$ and positions were initialized within $(-100,100)$. The experiment was performed in 50 dimensions. As a result the maximum possible distance between particles in the initial search space is 1414.214. This maximum distance 
is referred to as $\Delta_{\max }$ from this point forward. Reported results were capped at $\Delta_{\max }$ to prevent highly unstable parameter configurations from hindering the presentation of the results.

The measure of stability used in this paper is:

$$
\Delta_{m}(t+1)=\frac{1}{S_{m} \cdot n_{s}} \sum_{i=1}^{S_{m} \cdot n_{s}}\left\|\boldsymbol{x}_{i}(t+1)-\boldsymbol{x}_{i}(t)\right\|_{2} .
$$

where $S_{m} . n_{s}$ is the swarm size for each sub-swarm $m$. The sum of all $\Delta_{S_{m} . n_{s}}(t)$ 's is reported as $\Delta(t)$. The objective function used for each objective is

$$
C F(\boldsymbol{x}) \in U(-1000,1000),
$$

which was shown to be an effective objective function for stability analysis in [1].

The experiment was conducted over the following parameter region:

$$
w \in[-1.1,1.1], c_{1}+c_{2}+c_{3} \in(0,8], \text { and } \lambda \in[0,1],
$$

where $c_{1}=c_{2}=c_{3}$, with a sample point every 0.1 along $w, c_{1}+c_{2}+c_{3}$, and $\lambda$. A total of 1840 sample points from the region defined in equation (19) were used for each fixed $\lambda$. The results reported in section 5 are derived from 50 independent runs for each sample point.

\section{Experimental Results and Discussion}

This section presents the results of the experiments described in section 4 .

A snapshot of all parameter configurations' resulting stability measure values are presented in figures 2(a) to 3(e) for the last iteration of MGPSO with $\lambda$ set to, $0.0,0.1,0.2,0.3,0.4,0.5,0.6,0.7,0.8,0.9$, and 1.0 . The reported stability measures are the mean derived from the 50 independent runs.

The number of parameter configurations that empirically agree or disagree with the stable/unstable behavior predicted by the theoretically derived stability region of equation (16) is presented in table 1. Eight $\Delta_{m}$ based measurements are presented in table 1: the number of parameter configurations that are theoretically stable (TS) and unstable (TUS), the number of parameter configurations that where empirically stable (ES) and unstable (EUS), the number of parameter configurations that were found to be empirically stable despite the theory predicting unstable behavior (ES despite TUS), the number of parameter configurations that were found to be empirically unstable despite the theory predicting stable behavior (EUS despite TS), and lastly the percentage error and agreement between the theoretical derivation and the empirical findings. A parameter configuration is classified to be stable if the value of the recorded convergence measure of equation (17) is less than $\Delta_{\max }(d)$, and unstable if greater than or equal to $\Delta_{\max }(d)$, in accordance with the approach of Cleghorn and Engelbrecht [3]. 
Table 1: Empirical findings versus theoretical prediction

\begin{tabular}{|c||c|c|c|c|c|c|c|c|}
\hline$\lambda$ & TS & TUS & ES & EUS & ES despite TUS & EUS despite TS & Error & Agreement \\
\hline \hline 0 & 764 & 1076 & 759 & 1081 & 8 & 13 & $1.14 \%$ & $98.86 \%$ \\
\hline 0.1 & 781 & 1059 & 784 & 1056 & 12 & 9 & $1.14 \%$ & $98.86 \%$ \\
\hline 0.2 & 796 & 1044 & 798 & 1042 & 11 & 9 & $1.09 \%$ & $98.91 \%$ \\
\hline 0.3 & 809 & 1031 & 809 & 1031 & 12 & 12 & $1.3 \%$ & $98.7 \%$ \\
\hline 0.4 & 816 & 1024 & 819 & 1021 & 11 & 8 & $1.03 \%$ & $98.97 \%$ \\
\hline 0.5 & 817 & 1023 & 823 & 1017 & 14 & 8 & $1.2 \%$ & $98.8 \%$ \\
\hline 0.6 & 816 & 1024 & 816 & 1024 & 10 & 10 & $1.09 \%$ & $98.91 \%$ \\
\hline 0.7 & 809 & 1031 & 810 & 1030 & 9 & 8 & $0.92 \%$ & $99.08 \%$ \\
\hline 0.8 & 796 & 1044 & 798 & 1042 & 12 & 10 & $1.2 \%$ & $98.8 \%$ \\
\hline 0.9 & 781 & 1059 & 781 & 1059 & 10 & 10 & $1.09 \%$ & $98.91 \%$ \\
\hline 1 & 764 & 1076 & 760 & 1080 & 9 & 13 & $1.2 \%$ & $98.8 \%$ \\
\hline
\end{tabular}

As shown in figures 2(a) to $3(\mathrm{e})$ the shape and size of the regions empirically classified as stable is in-line with the theoretical prediction of equation (16). However, the effect of varying $\lambda$ is harder to see, which is not surprising given how similar the regions that the theoretical derivations predicts are, as illustrated in figure 1. The accuracy of the theoretical derivation can be more clearly seen in table 1. For all the tested $\lambda$ values, the theoretical prediction had an above 98.7\% accuracy. The accuracy reported was also stable across differing $\lambda$ values with the largest difference in accuracy reported being only $0.38 \%$.

It is evident from the presented results that the theoretically derived region for particle stability, as defined in equations (16) accurately reflect the parameter configurations needed for order-1 and order-2 stability of MGPSO.

\section{Conclusion}

This paper provided the first theoretical stability analysis of a multi-objective particle swarm optimization (PSO) variant. Specifically, this paper theoretically derived the order- 1 and order-2 stable regions for multi-guided PSO (MGPSO) using the minimal required modeling assumptions. The provided order-1 and order- 2 stable regions can be utilized by PSO practitioners to make an informed choice when selecting control parameters values for the MGPSO algorithm. Furthermore, the derived criteria for stability were validated, using the empirical method verified by Cleghorn and Engelbrecht [1], under which no simplifying modelling assumptions were placed on the MGPSO algorithm. Given the empirical validation, the theoretical derivation is an accurate representation of MGPSO stability criteria. 


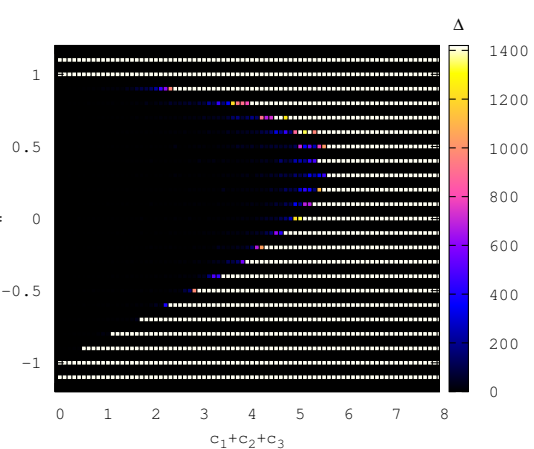

((a)) $\lambda=0.0$.

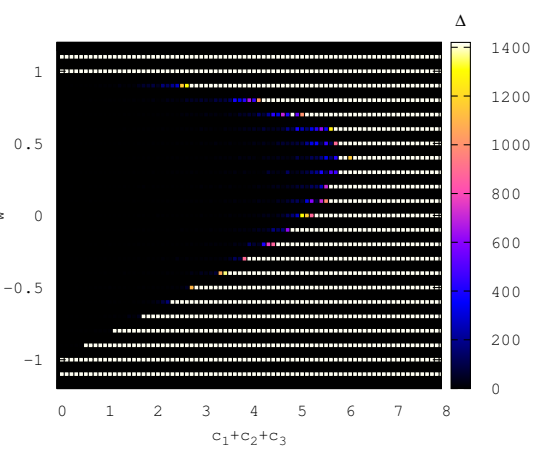

((c)) $\lambda=0.2$.

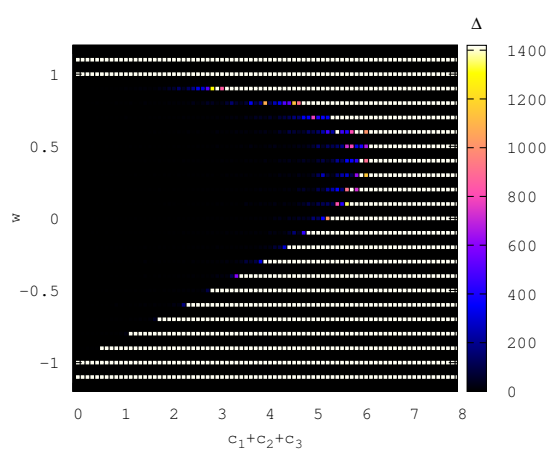

((e)) $\lambda=0.4$.

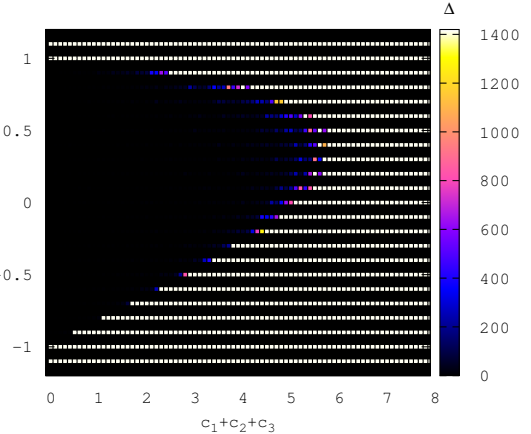

((b)) $\lambda=0.1$.

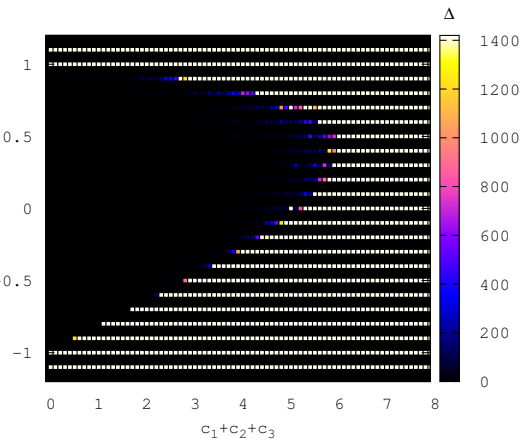

((d)) $\lambda=0.3$.

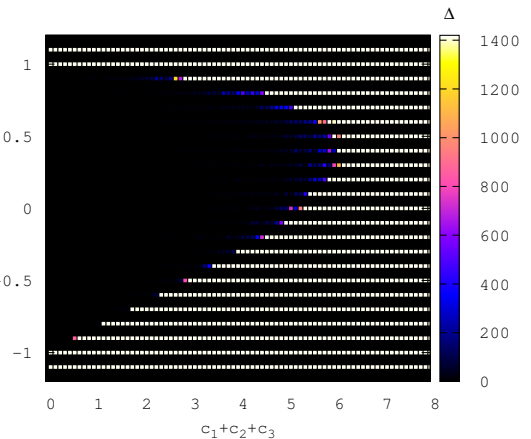

((f)) $\lambda=0.5$.

Fig. 2: MGPSO convergence results for $\lambda=0,0.1, \ldots, 0.5$ 


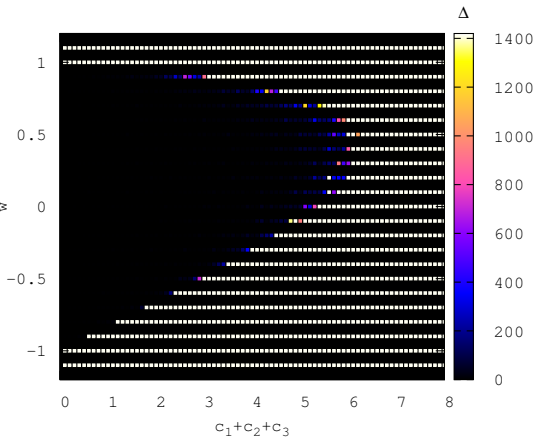

((a)) $\lambda=0.6$.

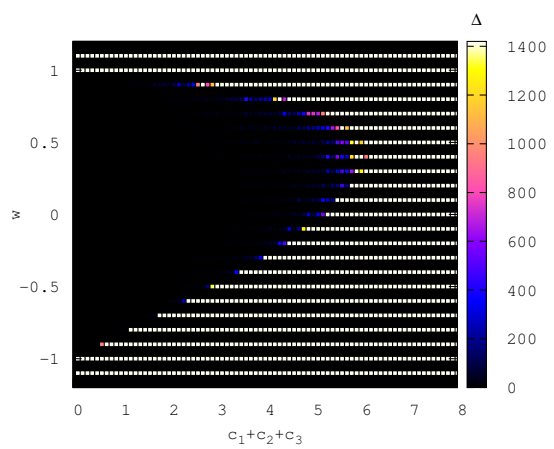

((c)) $\lambda=0.8$.

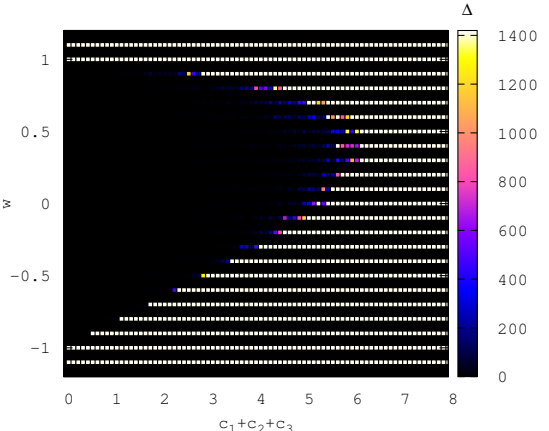

((b)) $\lambda=0.7$

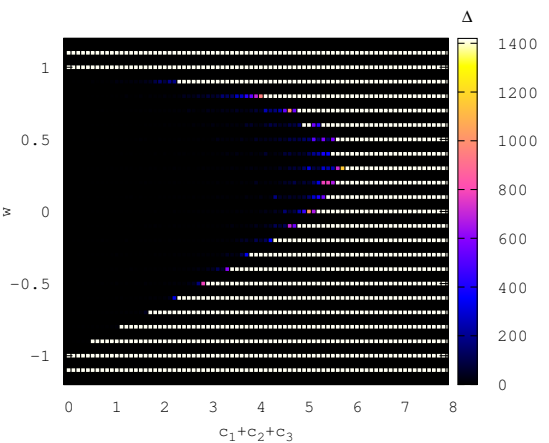

((d)) $\lambda=0.9$.

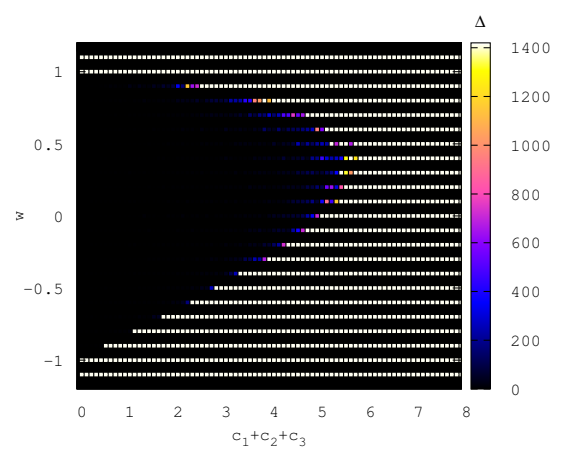

((e)) $\lambda=1.0$.

Fig. 3: MGPSO convergence results for $\lambda=0.6,0.7, \ldots, 1.0$ 


\section{References}

1. Cleghorn, C.W., Engelbrecht, A.P.: Particle swarm convergence: Standardized analysis and topological influence. In: Proceedings of International Swarm Intelligence Conference (ANTS), Swarm Intelligence. pp. 134-145. Springer International Publishing, Switzerland (2014)

2. Cleghorn, C.W., Engelbrecht, A.P.: Fully informed particle swarm optimizer: Convergence analysis. In: Proceedings of the IEEE Congress on Evolutionary Computation. pp. 164-170. IEEE Press, Piscataway, NJ (2015)

3. Cleghorn, C.W., Engelbrecht, A.P.: Particle swarm variants: Standardized convergence analysis. Swarm Intelligence 9(2-3), 177-203 (2015)

4. Cleghorn, C.W., Engelbrecht, A.P.: Particle swarm optimizer: The impact of unstable particles on performance. In: Proceedings of the IEEE Symposium Series on Swarm Intelligence. pp. 1-7. IEEE Press, Piscataway, NJ (2016)

5. Cleghorn, C.W., Engelbrecht, A.P.: Unified particle swarm optimizer: Convergence analysis. In: Proceedings of the IEEE Congress on Evolutionary Computation. pp. 448-454. IEEE Press, Piscataway, NJ (2016)

6. Cleghorn, C.W., Engelbrecht, A.P.: Particle swarm stability: a theoretical extension using the non-stagnate distribution assumption. Swarm Intelligence 12(1), 1-22 (2018)

7. Corne, D.W., Jerram, N., Knowles, J.D., Oates, M.L.: Pesa-ii: Region-based selection in evolutionary multiobjective optimization. In: Proceedings of the Genetic and Evolutionary Computation Conference. pp. 283-290. ACM Press, New York, NY (2001)

8. Deb, K., Agrawal, S., Pratap, A., Meyarivan, T.: A Fast Elitist Non-Dominated Sorting Genetic Algorithm for Multi-Objective Optimization: NSGA-II. In: Parallel Problem Solving from Nature. Lecture Notes in Computer Science. pp. 849-519. Springer, Berlin, Heidelberg (2000)

9. Kennedy, J., Eberhart, R.C.: Particle swarm optimization. In: Proceedings of the IEEE International Joint Conference on Neural Networks. pp. 1942-1948. IEEE Press, Piscataway, NJ (1995)

10. Nebro, A.J., Durillo, J.J., García-Nieto, J., Coello Coello, C.A., Luna, F., Alba, E.: SMPSO: A New PSO-based Metaheuristic for Multi-objective Optimization. In: Proceedings of the IEEE Symposium on MultiCriteria Decision-Making. pp. 66-73. IEEE Press, Piscataway, NJ (2009)

11. Parsopoulos, K.E., Vrahatis, M.N.: Particle swarm optimization method in multiobjective problems. In: Proceedings of the ACM Symposium on Applied Computing. pp. 603-607 (2002). https://doi.org/10.1145/508791.508907

12. Parsopoulos, K.E., Vrahatis, M.N.: Recent approaches to global optimization problems through Particle Swarm Optimization. Natural Computing 1(2), 235-306 (2002)

13. Reyes-Sierra, M., Coello Coello, C.A.: Improving PSO-Based Multiobjective Optimization Using Crowding, Mutation and Dominance. In: Evolutionary MultiCriterion Optimization. Lecture Notes in Computer Science. pp. 505-519. Springer, Berlin, Heidelberg (2005)

14. Scheepers, C.: Multi-guided Particle Swarm Optimization: A Multi-objective Particle Swarm Optimizer. Ph.D. thesis, Department of Computer Science, University of Pretoria, Pretoria, South Africa (2018)

15. Scheepers, C., Engelbrecht, A.P.: Multi-guide particle swarm optimization a multiswarm multi-objective particle swarm optimizer. Swarm Intelligence (under review) pp. 1-22 (2018) 
16. Shi, Y., Eberhart, R.C.: A modified particle swarm optimizer. In: Proceedings of the IEEE Congress on Evolutionary Computation. pp. 69-73. IEEE Press, Piscataway, NJ (1998)

17. Zhang, Q., Li, H.: IEEE Transactions on Evolutionary Computation. Natural Computing 11(2), 712-731 (2007)

18. Zitzler, E., Laumanns, M., Thiele.L: Spea2: Improving the strength pareto evolutionary algorithm. Tech. rep., Swiss Federal Instituteof Technology (ETH) Zurich (2001) 